\title{
Avaliação de Aspectos Quantitativos e Qualitativos da Dor na Fibromialgia
}

\section{Evaluation of the Quantitative and Qualitative Aspects of Pain in the Fibromyalgia Sydrome}

\author{
Simone Saltareli ${ }^{(1)}$, Débora Fernanda Amaral Pedrosa ${ }^{(2)}$, Priscilla Hortense ${ }^{(3)}$, \\ Fátima Aparecida Emm Faleiros Sousa ${ }^{(4)}$
}

\section{RESUMO}

Objetivo: Avaliar a percepção da dor na fibromialgia por meio de técnica metodológica quantitativa e qualitativa. Métodos: Foram avaliadas 30 pacientes mediante entrevista apreciada por meio de análise de conteúdo temática e do Instrumento de Descritores de Dor, porquanto para os dados resultantes foram calculados a média aritmética e o desvio-padrão para determinar quais descritores caracterizam a dor na fibromialgia. Resultados e Discussão: A análise de conteúdo resultou a construção de categorias de análise referentes às percepções de diagnóstico, motivações, doença, sentimentos, pensamentos e repercussões na qualidade de vida. Já o Instrumento de Descritores de Dor revelou que os descritores de maior atribuição na caracterização da dor foram: incômoda, que espalha, latejante, desconfortável e persistente, e os de menor atribuição foram: desgraçada, demoníaca, maldita, aterrorizante e assustadora. Os dois instrumentos mostraram tendência das pacientes em perceber e relatar a dor mais relacionada às características sensorial-discriminativas. Além disso, apresentaram dados relativos à importância do papel da família e do profissional de saúde no manejo da dor. Conclusão: Percebeu-se a necessidade de estimular a percepção e a expressão das pacientes em relação à dor, abarcando sua multidimensionalidade e, que o manejo da dor deve ser realizado levando-se em conta a tríade equipe de saúde, paciente e família, em face da complexidade do fenômeno.

Palavras-chave: fibromialgia, descritores de dor, análise de conteúdo.

\section{INTRODUÇÃO}

A fibromialgia é uma síndrome musculoesquelética crônica, caracterizada por dor generalizada de etiologia ainda não completamente esclarecida. É definida pelo Colégio Americano de Reumatologia (ACR) como dor

\begin{abstract}
Objective: Evaluate the perception of pain in the fibromyalgia through the quantitative and qualitative methodological technique. Method: A total of 30 patients were assessed through an interview analyzed by the thematic content and through the instrument Descriptors of Pain. Arithmetic mean and standard error were used to determine which descriptors better characterize the pain in the fibromyalgia. Results and Discussion: The result of the content analysis was the construction of categories of analysis regarding the perceptions of: diagnosis, motivation, disease, feelings, thoughts and repercussions on the quality of life. The Descriptors of Pain instrument revealed the descriptors of higher attribution in the characterization of pain were inconvenient, spreading, pulsating, uncomfortable and persistent and the descriptors with the lower attribution were miserable, demoniac, cursed, terrifying and frightening. The two instruments showed the patients' tendency in perceiving and reporting the pain regarding to the sensorial-discriminating characteristics. In addition, data related to the importance of the family's and the health professional's roles in managing the pain were presented. Conclusion: The need to stimulate the perception and expression of patients regarding the pain in its multidimensionality was perceived. It is concluded that the management of pain must be performed considering the complexity of the phenomenon in terms of the triad health team - patient - family.
\end{abstract}

Keywords: fibromyalgia, pain descriptors, content analysis.

generalizada há pelo menos três meses em combinação com a sensibilidade em 11 ou mais dos 18 pontos sensíveis à palpação localizados em várias partes do corpo, conhecidos como tender points ${ }^{(1)}$.

A complexidade de fatores envolvidos, tanto na questão do diagnóstico quanto na abordagem adequada pelos pro-

Recebido em 1/6/2007. Aprovado, após revisão, em 28/5/2008. Declaramos a inexistência de conflitos de interesse

Departamento de Enfermagem Geral e Especializada da Escola de Enfermagem de Ribeirão Preto da Universidade de São Paulo (USP)

1. Psicóloga, mestre da Escola de Enfermagem de Ribeirão Preto da USP.

2. Enfermeira, mestranda pela Escola de Enfermagem de Ribeirão Preto da USP.

3. Enfermeira, doutora pela Escola de Enfermagem de Ribeirão Preto da USP.

4. Professora-associada do Departamento de Enfermagem Geral e Especializada da Escola de Enfermagem de Ribeirão Preto da USP

Endereço para correspondência: Simone Saltareli, Rua Gildo Inácio, 124, 14096-670, Ribeirão Preto, SP, e-mail: ssaltareli@yahoo.com.br; ssaltareli@hotmail.com 
fissionais de saúde, mostra a necessidade de mais estudos que se proponham a ampliar o entendimento das temáticas vivenciadas por esses pacientes. De acordo com Masi, White e Pilcher ${ }^{(2)}$, modelos conceituais e metodologias de pesquisa ainda não se encontram suficientemente desenvolvidos para investigar algumas condições subjetivas ligadas à dor na fibromialgia.

Questionar a dor, avaliá-la, bem como a necessidade de mensurá-la, foi percebida no trabalho assistencial com esses tipos de pacientes, surgindo assim a motivação para o desenvolvimento de estudo que tivesse a proposta de fazê-lo de maneira mais precisa, fidedigna e clara, no intuito de, a partir disso, vislumbrar estratégias de atuação profissional mais adequadas na atenção e no manejo da dor percebida.

Para Goldenberg ${ }^{(3)}$, na realização de investigações de fenômenos complexos, a aplicação de técnicas metodológicas múltiplas possibilita melhor avaliação e compreensão deles. Assim, com variadas formas de coleta de dados, alcança-se acesso mais amplo à complexidade do fenômeno.

Com base nessas colocações, foram estabelecidos objetivos do estudo:

- Avaliar, por meio de uma técnica metodológica quantitativa e uma qualitativa, a percepção da dor em tratamento para fibromialgia;

- Investigar temáticas referentes à vivência do processo de adoecimento no contexto familiar, social e no relacionamento com os profissionais da saúde.

\section{PACIENTES E MÉTODOS}

Foram avaliadas 30 pacientes que, de acordo com os critérios de inclusão empregados, consentiram a participação no estudo. Elas tinham mais de 18 anos, não apresentavam condições metabólicas que pudessem alterar a percepção da dor e não apresentavam condições psiquiátricas ou neurológicas que impedissem a compreensão dos objetivos do estudo.

A aplicação de entrevista com 12 questões foi realizada com base no modelo proposto por Sturge-Jacobs ${ }^{(4)}$, na qual foram coletados dados sociodemográficos e, também, questionados aspectos da convivência social, familiar, relacionamento com as equipes de saúde, pensamentos e sentimentos que acompanham a vivência dessas questões. $\mathrm{O}$ conteúdo das entrevistas foi analisado segundo o método de análise de conteúdo proposta por Bardin ${ }^{(5)}$, especificamente a análise de conteúdo temática ${ }^{(6)}$.
Após a entrevista, foi aplicado o Instrumento de Descritores de Dor, proposto por Sant'Ana et al. ${ }^{(7)}$, que é constituído por 50 descritores de dor, visto que para cada um deles existe uma definição específica dada ao paciente no momento da aplicação do instrumento. É solicitado que o paciente atribua um valor numérico em uma escala com escores que variam de 0 a 6 , em que 0 é atribuído ao menor grau de caracterização da dor e 6 ao maior grau. As palavras utilizadas estão dispostas aleatoriamente e pertencem aos grupos sensoriais, avaliativos, afetivos e mistos, contemplando a multidimensionalidade do fenômeno doloroso.

\section{RESULTADOS}

Os dados sociodemográficos estão dispostos na Tabela 1 para ilustrar as características das pacientes participantes, sendo todas elas do sexo feminino, com idade média de 44,7 anos $( \pm 9,32)$.

TABela 1

Características Demográficas DAS PACIENTES AVALIADAS

\begin{tabular}{lc}
\hline Profissão & $73,3 \%$ \\
Remunerada & $26,7 \%$ \\
Não remunerada & \\
Procedência & $13,3 \%$ \\
$\quad$ Ribeirão Preto & $83,3 \%$ \\
Região & $3,3 \%$ \\
Outros estados & \\
Estado Civil & $73,3 \%$ \\
Amasiada/Casada & $13,3 \%$ \\
Divorciada/Separada & $10 \%$ \\
Solteira & $3,3 \%$ \\
Viúva & \\
\hline Número de filhos & $3,3 \%$ \\
Nenhum & $70 \%$ \\
Até 3 & $20 \%$ \\
4 ou 5 & $6,7 \%$ \\
Mais de 6 & \\
Escolaridade & $66,7 \%$ \\
$1^{\circ}$ grau incompleto & $13,3 \%$ \\
$1^{\circ}$ grau completo & $6,7 \%$ \\
$2^{\circ}$ grau incompleto & $13,3 \%$ \\
$2^{\circ}$ grau completo & \\
Religião & $30 \%$ \\
Evangélica & $60 \%$ \\
Católica & $10 \%$ \\
\hline Outras & \\
\hline & \\
\hline & \\
\hline
\end{tabular}

No que diz respeito às percepções das pacientes, a análise de conteúdo temático realizada originou o estabelecimento das seguintes unidades: diagnóstico, motivações, doença, sentimentos, pensamentos e repercussões na qualidade de vida. A Tabela 2 apresenta o panorama das categorias e subcategorias temáticas e a freqüência 
de ocorrência de cada uma delas. O amplo espectro de interpretações e relatos categorizados indica e reforça a complexidade do fenômeno doloroso, bem como a sua individualidade na expressão.

TABela 2

Categorias e subcategorias das temáticas ANALISADAS E SUAS FREQÜÊNCIAS DE OCORRÊNCIA NOS RELATOS DAS PACIENTES

\begin{tabular}{lc}
\hline Unidades temáticas & Freqüência de ocorrências \\
\hline Diagnóstico & 122 \\
Percepção dos sinais e/ou sintomas & 48 \\
Preocupações & 18 \\
Dificuldades de esclarecimento & 33 \\
Erros de diagnóstico & 18 \\
Dificuldades de acesso ao HC & 5 \\
Motivações & 188 \\
Perceção da equipe médica atual & 22 \\
Mudanças após a confirmação diagnóstica & 29 \\
Queixas de limitações físicas & 69 \\
Apoio familiar e/ou amigos & 49 \\
Apoio no trabalho & 19 \\
Doença & 238 \\
Percepção das causas e/ou influências & 65 \\
Recursos utilizados & 140 \\
Possibilidade de utilizar recursos próprios & 33 \\
Sentimentos & 105 \\
Percepção/identificação de sentimentos & 21 \\
Relação causas emocionais e sentimentos & 24 \\
Na relação familiar & 24 \\
Na relação conjugal & 28 \\
Luto & 8 \\
Pensamentos & 101 \\
Em relação ao futuro & 24 \\
Em relação às necessidades & 27 \\
Em relação ao tratamento & 5 \\
Idéias de perfeccionismo e/ou persistência & 21 \\
Em relação à doença em si & 24 \\
Repercussões na qualidade de vida & 87 \\
Familiares & 16 \\
Pessoais & 38 \\
Sociais/Trabalho & 29 \\
Sexuais & 4 \\
\hline &
\end{tabular}

A análise dos descritores de dor foi realizada por meio do cálculo das médias aritméticas e dos desvios-padrão, calculados para cada um dos descritores. Em seguida, foi estabelecido um ranking, no qual foram dispostos os descritores de maior atribuição e os de menor atribuição. As Tabelas 3 e 4 apresentam, respectivamente, estes dados, mostrando os cinco descritores mais significativos.

\section{DISCUSSÃO}

$\mathrm{Na}$ categoria diagnóstico, foram sistematizados os dados relativos ao percurso realizado pelas pacientes. A procura para
TABela 3

Descritores de MAior ATRIBUição NA CARACTERIZAÇ̃̃O DA DOR CRÔNICA DAS PACIENTES AVALIADAS

\begin{tabular}{|lccc|}
\hline Ordem de posição & Médias aritméticas & Desvios-padrão & Descritores \\
\hline 1 & 5,6 & 0,72 & Incômoda \\
\hline 2 & 5,53 & 1,28 & Que espalha \\
\hline 3 & 5,5 & 0,82 & Latejante \\
\hline 4 & 5,5 & 0,94 & Desconfortável \\
\hline 5 & 5,5 & 1,14 & Persistente \\
\hline
\end{tabular}

Tabela 4

DESCRITORES DE MENOR ATRIBUICC̃̃O NA CARACTERIZAÇ̃̃o DA DOR CRÔNICA DAS PACIENTES AVALIADAS

\begin{tabular}{lccc}
\hline Ordem de posição & Médias aritméticas & Desvios-padrão & Descritores \\
\hline 1 & 3,2 & 2,54 & Desgraçada \\
\hline 2 & 3,4 & 2,74 & Demoníaca \\
3 & 3,7 & 2,48 & Maldita \\
\hline 4 & 3,77 & 2,24 & Aterrorizante \\
5 & 3,8 & 2,2 & Assustadora \\
\hline
\end{tabular}

realização e/ou confirmação do diagnóstico foi de um a cinco anos para $50 \%$ das pacientes, entre cinco e dez anos para $20 \%$ e de mais de dez anos para $3,3 \%$. O número de médicos consultados foi de até três para $46,7 \%$ das pacientes, até cinco para $23,3 \%$, até dez para $3,3 \%$, e $26,7 \%$ das pacientes referiram ter consultado vários médicos não conseguindo precisar a quantidade destes. Os serviços de saúde em que foram realizados os atendimentos foram até três serviços para $90 \%$ das pacientes e de mais de três para $10 \%$, havendo relatos de procura tanto por médicos do Sistema Único de Saúde (SUS) quanto, também, por convênios e médicos particulares. Em relação à freqüência atual de procura por serviços de saúde por causa das crises álgicas, as pacientes relataram que $63,3 \%$ procuram o serviço pelo menos uma vez por mês, $13,3 \%$ procuram duas vezes, $6,7 \%$ procuram três vezes ao mês e $16,7 \%$ chegam a procurar quatro vezes ou mais. Além disso, apresentam dados referentes à percepção de sinais e/ou sintomas associados, a preocupação gerada tanto pela espera do diagnóstico quanto pelas preocupações relacionadas a dúvidas ainda não esclarecidas, como exemplifica o relato a seguir:

"Ah, eu acho que sim né, porque eu tinha até medo de ser câncer, por exemplo. Falava, gente, essa dor, essa dor, eu devo tá com alguma coisa. Ai a doutora falou assim 'pode ficar tranqüila, não tem nada a ver'." 
As dificuldades de esclarecimento também foram analisadas e percebeu-se que as características pessoais tinham grande influência, especialmente no que se refere a timidez e vergonha de questionar.

Erros diagnósticos foi uma outra unidade temática importante em relação ao diagnóstico, pois revelou condição amplamente discutida na literatura. Heymann ${ }^{(8)}$ discute a falta de atenção recebida até hoje por pacientes com fibromialgia nos consultórios, o que em parte deve-se à falta de conhecimento do profissional e também pela falta de disposição em aprofundar-se no assunto.

"Foi por causa de dor..., ai o doutor Paulo falou que era má circulação. Falou 'quem sabe se fazendo cirurgia das pernas não acaba essa dor'. Então a gente faz de tudo pra acabar com a dor, né? O que a gente quer é tirar as dores. Ai eu fiz a cirurgia. Mas não virou nada, ela não inchava, agora começou a inchar."

A dificuldade de chegar ao hospital de nível terciário, onde foi realizado o estudo, também foi comentado pelas pacientes, especialmente pela demora de encaminhamento.

$\mathrm{Na}$ categoria motivações, foram analisadas a percepção da equipe médica atual, com relatos de percepção positiva e também percepção negativa. Essa unidade de análise mostra a importância da relação do paciente com o profissional de saúde, e que a associação de estratégias é necessária, bem como a monitoração e a eficácia justificando a continuidade do manejo ${ }^{(9)}$.

Essa percepção também fica clara na unidade referente à mudança percebida após a confirmação diagnóstica, indicando que as preocupações relacionadas à dor surgem como algo importante, que são desvencilhadas por meio do conhecimento da doença, das informações advindas desse processo e da preparação para buscar efetividade no manejo.

"Faz. Faz porque você sente dor, sem saber o que é, te causa preocupação. Você sabendo o que é, você sabe o que é 'eu tô com dor porque eu tenho isso. Então vou sentir essa dor, vai ser freqüente, vai ser sempre'. A médica já me falou que não tem cura, é uma melhora. Vai ser sempre assim, hoje tá bom, amanhã vai doer, é assim que eu vou ter de acostumar."

O paciente que desconhece a doença que tem pede por um diagnóstico na tentativa de evitar o mal-estar crônico que surge com o adoecimento ${ }^{(10)}$.

As queixas de limitações físicas somaram montante considerável, expondo as principais queixas que estão descritas na literatura em diversos estudos ${ }^{(1,11-13)}$.

$\mathrm{O}$ apoio familiar, de amigos e no trabalho, apresentouse de maneira bastante variada na percepção das pacientes, mostrando, em muitos casos, a existência de apoio, e, em outros, um total descaso, dificultando o manejo.

"E eu já fico nervosa, en falo 'eu já tô com dor, e vocês ainda ficam tirando o sarro de mim? Eu tô com dor, não tô brincando, tô falando a verdade'. Eles acham que porque eu tô ali, lavando uma roupa, fazendo uma comida, limpando uma casa, que é mentira minha que eu tô com dor."

A diminuição da capacidade funcional é uma das principais conseqüências da fibromialgia, pois tem implicações sociais, laborais e também familiares ${ }^{(14)}$.

$\mathrm{Na}$ categoria doença, a percepção das causas e influências contou com aspectos tanto físicos quanto emocionais, confirmando mais uma vez dados trazidos pela literatura ${ }^{(15)}$.

Outros temas foram relacionados aos recursos utilizados, subdivididos em pessoais ( 52 ocorrências), medicamentosos (43 ocorrências), religiosos (20 ocorrências), sociais (14 ocorrências) e familiares (11 ocorrências). Percebe-se aqui a preponderância de recursos pessoais e medicamentosos. A possibilidade de utilizar recursos próprios contou também com opiniões discordantes, em que algumas pacientes perceberam essa possibilidade, nos aspectos físicos e emocionais e outras não perceberam tal possibilidade.

"Pra melhorar mais, no momento eu ainda não encontrei. Não encontrei ainda não, mas não vou deixar de procurar não, vou estar sempre."

$\mathrm{Na}$ categoria sentimentos, a identificação de sentimentos foi relatada por 21 pacientes, sendo percebida certa dificuldade na expressão destes, tendo a entrevistadora questionado várias vezes para obter resposta. A carga emocional presente é perceptível e ilustrada pelo relato seguinte:

"Nossa é só raiva e ódio. Só raiva e ódio. Eu tenho vontade de pôr fogo em tudo, acabar com o mundo, com a minha vida, nossa! Só penso isso, só. Sempre pensei, que eu falei pra você que eu tentei até suicidar, né? Eu penso isso.”

Os dados explicitados aqui condizem com os encontrados na literatura, que reportam a sentimentos de menos valia, baixa auto-estima e pessimismo ${ }^{(16-18)}$.

O estabelecimento de relação entre causas emocionais e sentimentos resultantes também fizeram parte da análise, principalmente em relação à veracidade da dor sentida.

"Ah, eu sinto que tá duvidando de mim, duvidando das minhas dores. Porque eu acho que ninguém fala uma coisa dessas que não tá sentindo. Tá certo que tem coisas da cabeça mesmo, mas tem coisas que não é."

Os sentimentos na relação familiar e na relação conjugal expressam o reflexo do amparo recebido, variando bastante, de acordo com o tipo de apoio percebido, quando percebido. 
Alguns relatos relacionados ao luto também fizeram parte da descrição da percepção dos sentimentos pelas pacientes.

$\mathrm{Na}$ categoria pensamentos, foram priorizados relatos que abordassem o entendimento racional das pacientes em relação aos questionamentos. Assim, os pensamentos em relação ao futuro indicaram projeções quanto às possibilidades de piora e de melhora da doença e sua influência no nível de limitações percebidas. A variabilidade nos níveis de dor tem papel importante na contribuição para essa perspectiva, fazendo que a paciente perceba a dor de maneira alternada em períodos de melhora e de piora, dando margem para idéias de agravamento e/ou melhora.

Os pensamentos em relação às necessidades refletem a visão das pacientes sobre o que seria necessário, e de ajuda para um manejo mais adequado.

"Ah, acho que na minha casa é falta de amor, né? Acho que meu marido é muito... ah, sei lá, eu acho que ele vê que eu tô precisando de médico, ele ao invés de me ajudar, acho que ele se afasta de mim, sabe, e não conversa comigo muito, só conversa o necessário comigo. Não sei se eu tô te respondendo certo."

Em relação ao tratamento, as pacientes mostram dados relativos à crença no tratamento e na efetividade deste, o que se relaciona novamente com a atitude do profissional de saúde diante dessa questão. São necessários, assim, a associação de recursos terapêuticos para uma resposta, senão mais efetiva, que consiga fazer que outras possibilidades possam ser encontradas em conjunto na relação das pacientes com a equipe de saúde como um todo ${ }^{(19,20)}$.

As idéias de perfeccionismo e/ou persistência indicam característica de pensamento investigada em outros estudos também e revelam como as pacientes tentam resistir à dor, mantendo suas atividades ${ }^{(21)}$.

A última unidade dessa categoria apresenta os pensamentos em relação à doença em si, de que forma as pacientes percebem a doença, relatando conformismo em alguns casos e dificuldade de acreditar no diagnóstico no outro extremo, em razão de ausência de resultados confirmados por exames laboratoriais.

"Ah, é difícil. Sabe porque, se tivesse alguma coisa que te mostrasse o que é essa fibromialgia, você já sabia. 'Não é porque saiu no diagnóstico, eu tenho que ter', você entendeu? Mas é uma coisa que não sai em lugar nenhum, e é uma dor... Como que eu sinto as dores? Porque não sai, não dá pra entrar na cabeça da gente. Como que existe essa dor, essas doenças, essas coisas ruins que a gente sente no corpo? Pontada, agulhada."

A categoria repercussões na qualidade de vida mostra o impacto percebido com a doença e os prejuízos impostos no âmbito familiar (com alterações na dinâmica e nas relações da família), pessoal (relacionado principalmente com a dependência gerada pelas limitações e mudanças no estado de humor), sociais e de trabalho (com diminuição da produtividade e das atividades de lazer) e sexuais (interferindo no apetite sexual das pacientes). $\mathrm{O}$ impacto na qualidade de vida foi discutido em vários estudos e os dados confirmam os encontrados neste estudo ${ }^{(13,17,18,22)}$.

A análise do Instrumento de Descritores de Dor revelou que os descritores de maior atribuição na caracterização da dor foram: incômoda, que espalha, latejante, desconfortável e persistente. Sendo estes os cinco primeiros descritores utilizados pelas pacientes na caracterização da dor, o aspecto sensitivo ganha destaque na percepção que essas pacientes têm de sua própria dor. Dados da literatura confirmam esses resultados, tanto no que se refere ao aspecto orgânico quanto cultural da percepção da dor na fibromialgia, segundo Giesecke et al. ${ }^{(13)}$ e Quintner, Buchanan e Taylor ${ }^{(23)}$.

Os cinco descritores de menor atribuição na caracterização da dor foram: desgraçada, demoníaca, maldita, aterrorizante e assustadora. A análise aqui denota forte componente religioso, no que diz respeito aos três primeiros descritores. O fato de todas as pacientes entrevistadas referirem ter participação em organizações religiosas surge como hipótese para justificar o motivo pelo qual justamente esses três descritores terem recebido menor atribuição.

Com base na análise dos dados obtidos, acredita-se ser importante para a eficácia do tratamento que a ênfase das intervenções devam embasar-se na tríade equipe de saúde, paciente e família, de modo que, inter-relacionando essas três unidades, possam abarcar o fenômeno de maneira mais completa, trazendo a família para a realidade da doença, esclarecendo dúvidas, fazendo trabalho de orientação e busca pela reorganização e o fortalecimento das relações familiares em função da otimização no manejo da dor.

Além das intervenções relativas à dinâmica da relação do paciente com os demais envolvidos em uma relação mais direta, encontram-se também possibilidades para melhor compreensão até mesmo do paciente em relação à dor, incentivando outras formas de percepção e expressão da dor referida, o que pode, em última instância, facilitar a compreensão do fenômeno para eles mesmos, assim como para os profissionais e demais indivíduos que fazem parte de seu convívio.

Há necessidade de continuidade de pesquisas que possam cada vez mais somar estratégias que possam alcançar o alívio do sofrimento físico, psíquico, social e familiar gerado pela doença. 


\section{REFERÊNCIAS}

1. Wolfe, F, Smythe, HA, Yunus, MB, et al.: The American College of Rheumatology 1990, criteria for the classification of fibromyalgia: report of the multicenter criteria committed. Arthritis Rheum 33: 160-72, 1990.

2. Masi AT, White KP, Pilcher JJ: Person-centered approach to care, teaching, and research in fibromyalgia syndrome: Justification from biopsychosocial perspectives in populations. Seminars in Arthritis Rheum 32(2): 71-93, 2002.

3. Goldenberg M: A arte de pesquisar: como fazer pesquisa qualitativa em ciências sociais. 4.ed. Rio de Janeiro-São Paulo: Record, 2000.

4. Sturge-Jacobs M: The experience of living with fibromyalgia: confronting an invisible disability. Res Theory Nur Pract 16: 19-31, 2002

5. Bardin L: Análise de conteúdo. Lisboa: Edições 70; 1979. 226p.

6. Minayo MC: $\mathrm{O}$ desafio do conhecimento: pesquisa qualitativa em saúde. 7.ed. São Paulo-Rio de Janeiro: Hucitec-Abrasco, 2000.

7. Sant'ana RPM, Pereira LV, Saltareli S, Sousa FF: Chronic pain descriptors: a psychophisical study. XX Annual Meating of the International Society for Psychiphisics - Fechner Day 2004. Universidade de Coimbra, Portugal, 2004.

8. Heymann RE: O papel do reumatologista frente à fibromialgia e à dor crônica musculoesquelética. Rev Bras Reumatol 46(1): $1,2006$.

9. Fitzcharles M, Almahrezi A, Shir Y: Pain: understanding and challenges for the rheumatologist. Arthritis Rheum 52: 3685-92, 2005 .

10. Ehlers L: Pain and new cultural diseases. Endod Dent Traumatol 15: 193-7, 1999.

11. Martinez JE, Atra E, Ferraz MB, Silva PSB: Fibromialgia: aspectos clínicos e socioeconômicos. Rev Bras Reumatol 32: 225-30,1992.
12. Gracely RH, Grant MAB, Giesecke T: Evoked pain measures in fibromyalgia. Best Pract Res Clin Rheumatol 17: 593-609, 2003.

13. Giesecke, T, Williams DA, Harris RE, et al.: Subgrouping of fibromyalgia patients on the basis of pressure-pain thresholds and psychological factors. Arthritis Rheum 48(10): 2916-22, 2003.

14. Martinez JE, Panossian C, Gavioli F: Estudo comparativo das características clínicas e abordagem de pacientes com fibromialgia atendidos em serviço público de reumatologia e em consultório particular. Rev Bras Reumatol 46: 32-6, 2006.

15. Arnold LM, Hudson JI, Hess EV: Family study of fibromyalgia. Arthritis Rheum 50(3): 944-52, 2004.

16. Cavaliere MLA: Efetividade de um programa multidisciplinar de tratamento da fibromialgia. [Dissertação]. Universidade Estadual do Rio de Janeiro, 2002.

17. Berber JSS, Kupek E, Berber SC: Prevalência de depressão e sua relação com a qualidade de vida em pacientes com síndrome da fibromialgia. Rev Bras Reumatol 45(2): 47-54, 2005.

18. Hassett AL, Cone JD, Patella SJ, Sigal LH: The role of catastrophizing in the pain and depression of women with fibromyalgia syndrome. Arthritis Rheum 43(11):2493-500, 2000.

19. Gallinaro AL: Avaliação da associação entre fibromialgia e lesão por esforço repetitivo em metalúrgicos de Guarulhos. [Tese] Faculdade de Medicina da Universidade de São Paulo, 1999.

20. Harris RE, Williams DA, Mclean SA, et al.: Characterizations and consequences of pain variability in individuals with fibromyalgia. Arthritis Rheum 52(11): 3670-4, 2005.

21. Weidebach WFS: Fibromialgia: evidências de um substrato neurofisiológico. Rev Assoc Med Bras 48: 291, 2002.

22. Araújo RL: Fibromialgia: construção da realidade na formação dos médicos. Rev Bras Reumatol 46: 50-60, 2006.

23. Quintner J, Buchanan MC, Taylor A: Signification and pain: a semiotic reading of fibromyalgia. Theo Med 24: 345-54, 2003. 